\title{
Verification of temperature distribution in the system for pad welding by means of infrared thermography
}

\author{
by I. Szczygieł ${ }^{*}$ A. Fic ${ }^{*}$, T. Kruczek* and A. Sachajdak*
}

*Institute of Thermal Technology, Silesian University of Technology, Konarskiego 22, 44-100 Gliwice, Poland

\section{Abstract}

The heat transfer in the system for pad welding of plates is considered. Particularly, the presence of air gap between the padded material and technological stand is investigated. The air gap appears due to the extremely high temperatures in the vicinity of the welding pool, which involve a deformation of the padded plate. The heat resistance of the air gap causes a strong decrease of the heat flux transferred from the welding pool. Intensity of this heat transfer has the essential meaning for achieving the welding pad of good quality. The presented analysis is the initial step of investigations referred to the estimation of the gap heat resistance using inverse procedure based on temperature measurements. In the paper, the mathematical model of the heat transfer in the system considered is formulated. The basic assumptions are described and discussed. The problem is solved numerically. The commercial code FLUENT is used for this purpose. Results of calculations are verified with the usage of infrared thermography.

\section{Introduction}

Pad welding is often contemporary used in practice to build up components back to their original thickness and integrity or to improve properties of surfaces working in extremely hard conditions. The technology can be applied to produce components of high surface hardness or abrasion, as well as components, which are able to withstand very high temperatures. For this purpose, the surfaces of such components are padded by materials of proper quality, e.g. ceramets or superalloys. Blades of fans working in erosion conditions can be referred as examples of this kind parts of machines.

In the paper the GMA (gas metal arc) technology is investigated (Fig. 2). The deposit is delivered to the base material in the inert gas shield. Melting the wire is accomplished by the electric arc acting between two electrodes. First is the base material (cathode), while the wire constitutes the second one (anode). The electric potential between the electrodes causes the current flow, which generates thermal energy in the partially ionised gas. It creates also magnetic field interacting with the current field to induce electromagnetic forces, which accelerate the plasma flow towards the workpiece surface. The high temperature plasma arc melts the tip of the wire, which involves formation of molten droplets driven through the plasma into the welding pool.

The heat transfer in the system: padding weld, padded material, air gap, welding bench and environment is generally unsteady and has 3D form. It is affected both by convection and radiation, as well as by geometrical and physical properties of the components. The heat transfer in the welding pool is very complicated. The deposit is delivered to welding pool created on the base material in a form of molten droplets. It makes the surface of the welding pool unstable. Besides, thermal phenomena in the vicinity of the welding pool are very complex. They are affected also by electrical and electromagnetic phenomena in the plasma arc. In the system considered, the air gap occurs between the padded material and the welding bench as the result of thermal deformation of the padded plate due to extremely high local temperatures in the vicinity of the welding pool. The heat resistance of the air gap influences strongly the heat transfer in the considered system. This heat resistance is difficult to evaluate, as it depends both on radiative, convective and conductive heat transfer in the gap. This resistance is generally space depended. The heat transfer problem considered is strongly nonlinear, as it coupled with other mentioned above phenomena and due to existence of the radiative heat transfer in the system. Thus, the procedure which allows to determine the heat resistance of the considered air gap is a very challenging task.

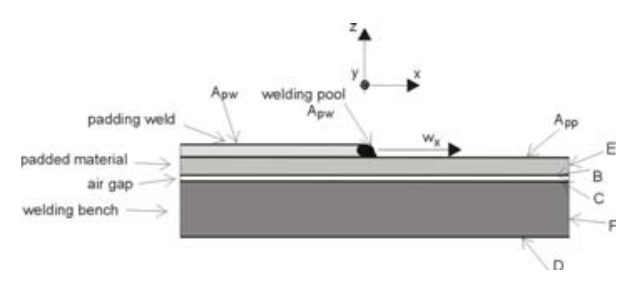

Fig. 1. Cross section of the pad welding system

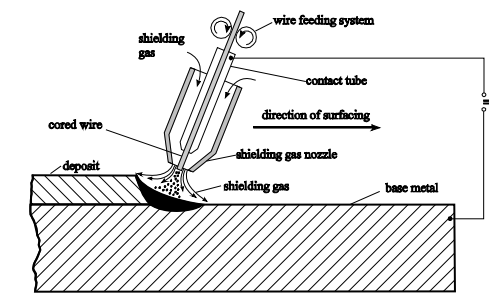

Fig. 2. Scheme of a system for GMA pad welding

\section{Formulation and solution of the problem}

The vertical cross section of the assumed 3D computational domain is shown in Fig. 1. It crosses the currently made layer of the padded weld. Taking into account assumptions mentioned above, the steady state temperature distribution $T(x, y, z)$ is described within the following layers of the system, i.e. the padding weld, padded material, and the welding bench, by the energy equation of the form: 


$$
-w_{x} c \rho \frac{\partial T}{\partial x}=\frac{\partial}{\partial x}\left(k_{x} \frac{\partial T}{\partial x}\right)+\frac{\partial}{\partial y}\left(k_{y} \frac{\partial T}{\partial y}\right)+\frac{\partial}{\partial z}\left(k_{z} \frac{\partial T}{\partial z}\right)+q_{v}
$$

where $k_{x}, k_{y} k_{z}$ are the directional heat conductivities, $c$ stands for the heat capacity, $\rho$ denotes density and $q_{v}$ is the heat source term. In the considered coordinate system attached to the welding pool, components of the system move in the $-x$ direction, what involves negative sign of the left hand site of Eqn (1).

Assumed boundary conditions have forms:

- convection and radiation on the surfaces $A_{w p}, A_{p p}$ and $D$,

- heat resistance in the air gap,

- continuity conditions for the temperature and heat flux on the welding pad - padded material boundary,

- known temperature along surfaces $E, F$ and $A_{w p}$ (the welding pool surface where the material of the welding pad is delivered),

- thermal insulation along remaining boundaries,

- Stefan's boundary conditions along the phase change interface $\Gamma_{p h}$, which unknown location has to be determined.

The problem defined above is solved using the commercial package FLUENT. The possessed in-house code of the finite element method (FEM) can also be applied for this purpose and its recalling within the inverse procedure will be more convenient. However, non-conformal meshes available for FLUENT can not be used in the FEM code. It makes meshes prepared for the FEM code much larger than non-conformal meshes for FLUENT. As a result, it allows to safe significantly computational time applying FLUENT, although FLUENT package is essentially directed for solving fluid flow problems.

\section{Results of calculations}

Results presented in this subsection were performed for constant value of the heat resistance of the air gap. This resistance is equal to $R=0.004 \mathrm{~m}^{2} \mathrm{~K} / \mathrm{W}$

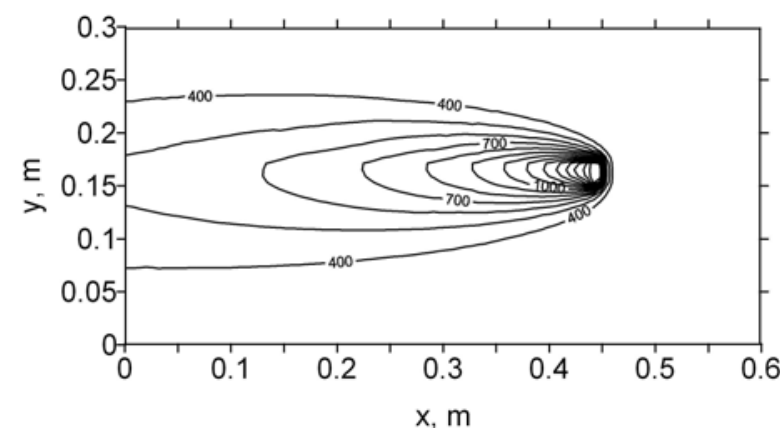

Fig. 3. 2D plot of the upper surface temperature, $\mathrm{K}$

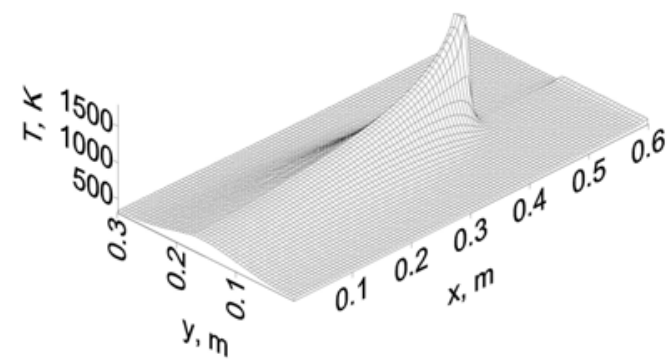

Fig. 4. 3D plot of the upper surface temperature, $\mathrm{K}$

\section{Experimental verification}

The numerical simulation was verified with the usage of infrared mapping. The exemplary map is presented in the figure 5. The comparison with the computations is shown in the figure 6.

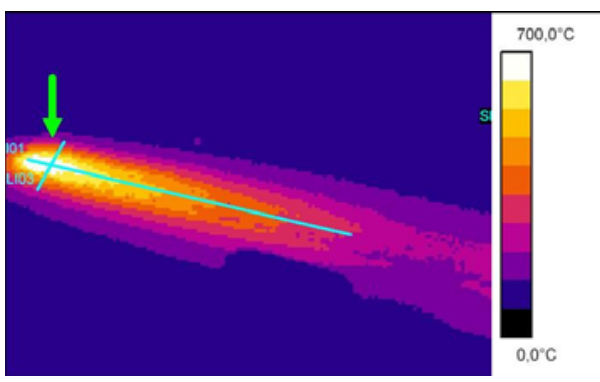

Fig 5. Exemplary map of temperature

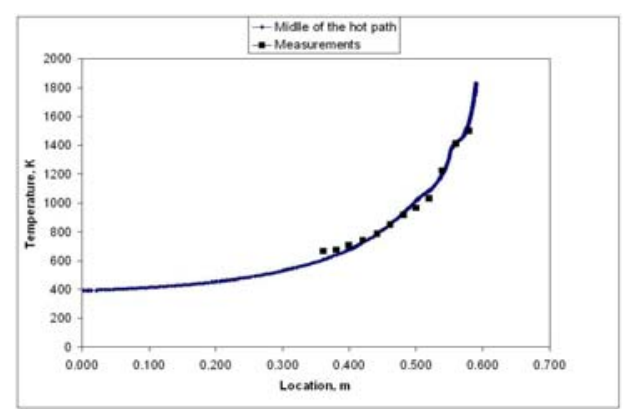

Fig 6. Comparison of experiment and simulation

\section{REFERENCES}

[1] Klimpel A. et al., Abrasion resistance of GMA metal cord wires surfaced deposits, Journal of Materials Processing Technology, 164-165, pp. 1056-1061, 2005.

[2] Klimpel A. and Mazur M., Podręcznik spawalnictwa (in Polish), Wyd. Pol. SI., 2004.

[3] Pilarczyk J. et al., Welding Technology, (in Polish), WNT Warsaw, 2003. 